bilirubinometer is somewhat more effective than clinical screening. If both transcutaneous index and clinical acumen were used simultaneously there would be considerably fewer false-positive screens.

\section{Discussion}

Previous studies evaluating the transcutaneous bilirubinometer have been carried out in Japan by Yamanouchi et al., ${ }^{2}$ and in the USA by Hegyi et al. ${ }^{3}$

Hegyi et al. ${ }^{3}$ obtained a correlation coefficient of 0.77 in forehead measurements on 43 white term infants on no treatment compared with our figure of $0 \cdot 65$. Yamanouchi ${ }^{2}$ obtained a much more significant correlation coefficient of 0.877 in 40 measurements in the forehead area. Differences in methods of serum bilirubin analysis must be considered when noting the disparity, as Hegyi et al. ${ }^{3}$ used a spectrophotometric technique and Yamanouchi ${ }^{2}$ used the Amerioan optical bilirubinometer and an alkali AZO bilirubin me hod.

Our correlation coefficient of 0.76 in 24 term measurements during phototherapy treatment was in fact better than in the untreated group $(\mathrm{r}=0.65)$. Yamanouchi ${ }^{2}$ however, found poor correlation of total serum bilirubin and transcutaneous index in term and preterm babies on phototherapy.

Since an individual transcutaneous index did not predict a serum bilirubin level accurately, we could not agree with Yamanouchi $^{2}$ that the instrument could serve as a viable alternative to traditional invasive procedures used to determine serum bilirubin concentration.

Evaluation of the screening potential of the instrument has not been previously undertaken. Our study (Table) confirms its va ue as a screening method in term babies on no treatment. There was an inadequate complement of measurements to est $a b l i-h$ index screening criteria in preterm infants.

Yamanouchi and Hegyi concluded from their studies $^{2} 3$ that the instrument was precise and accurate and our findings confirmed this. Yamanouchi et $a .^{2}{ }^{2}$ found that precision decreased at increasing serum bilirubin concentrations but there was no evidence of this in our data.

During the study we assessed a subgroup of 4 babies with rhesus haemolytic disease all of whom required exchange transfusion. There was a very poor correlation in the pre-exchange bilirubin level and the transcutaneous index measurement. After exchange transfusions the reduction in the level of serum bilirubin was not associated with any change in the transcutaneous index. This would agree with the opinion of Yamanouchi et al. ${ }^{2}$ that the transcutaneous bilirubin measurement is contraindicated in rhesus haemolytic disease and in babies after exchange transfusion.

The transcutaneous bilirubinometer has a useful role in a busy maternity hospital with a frequent turnover of junior doctors and nursing staff. Its role here is to act as an adjunct to clinical screening in determining which term babies are likely to have serum bilirubin concentrations greater than 250 $\mu \mathrm{mol} / \mathrm{l}$. It does not have a role as an alternative to serum bilirubin determination in all cases.

\section{References \\ 1 Lucey J F, Nyborg E, Yamanouchi I. A new device for transcutaneous bilirubinometry. Pediatr Res 1980; 14: 604. \\ 2 Yamanouchi I, Yamauchi Y, Igarashi I. Transcutaneous bilirubinometry: preliminary studies of non-invasive transcutaneous bilirubin meter in the Okayama National Hospital. Pediatrics 1980; 65 : 195-202. \\ ${ }^{3}$ Hegyi T, Hiatt M I, Indyk L. Transcutaneous bilirubino- metry. I. Correlations in term infants. J Pediatr 1981; 98 : 454-7.}

Correspondence to Dr Margaret Sheridan-Pereira, National Maternity Hospital, Holles Street, Dublin 2.

Received 30 March 1982

\title{
Uncombable hair: a condition with autosomal dominant inheritance
}

\section{B GARTY, A METZKER, M MIMOUNI, AND I VARSANO}

Department of Paediatrics ' $B$ ' and Paediatric Dermatology Clinic, Beilinson Medical Centre, Petah-Tiqva, and Tel-Aviv University, Sackler School of Medicine, Israel

SUMMARY Uncombable hair is a familial abnormality of scalp hair structure which affects children and lessens as they grow older. It is suggested that the mode of inheritance of the condition is autosomal dominant.
In 1973 Stroud and Mehregan ${ }^{1}$ reported a patient with unusual hair which they termed 'spun glass hair'. In the same year Dupré et al. ${ }^{2}$ described a similar hair abnormality which they called the 'uncombable hair syndrome' or 'pili trianguli et canaliculi' according to the ultrastructural finding. 
Recently, Ferrando et al. ${ }^{3}$ stressed that a longitudinal groove was the only characteristic morphological finding of this abnormality and proposed the name 'pili canaliculi'.

Since it only affects children, it seems surprising that the anomaly has not been described in paediatric journals. This paper describes a typical familial

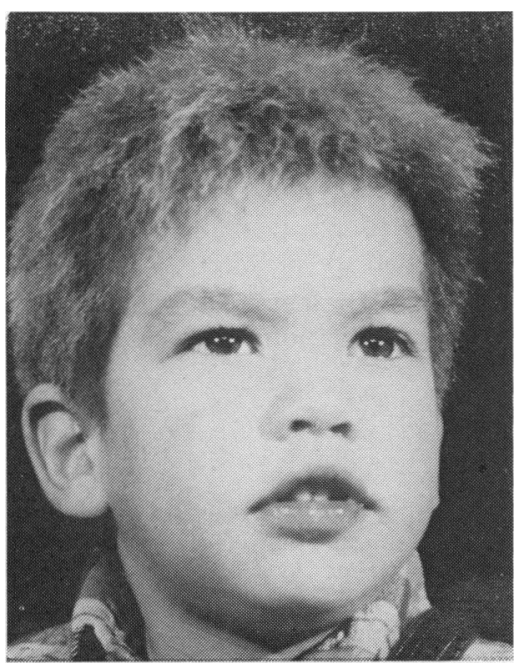

Fig. 1 General appearance of the hair of the patient. case, suggesting an autosomal dominant mode of inheritance.

\section{Case report}

A 2-year-old boy, the only son of unrelated, healthy, Jewish Ashkenazi parents, was normally delivered after an uneventful pregnancy. His weight, height, and head circumference, and his psychomotor development are in accordance with his chronological age. He has coarse facial features, a hoarse voice, and bilateral inner epicanthal folds. His scalp hair has a peculiar frizzy structure; the hair bundles are disposed in different directions and cannot be arranged by combing (Fig. 1). The hair is dry, coarse, thick, and not fragile; its colour is reddishblond with a characteristic shine. The eyebrows are thick and of a similar colour. The skin, eyelashes, velum hair, nails, and teeth are normal. The rest of the physical examination is unremarkable.

The peculiar appearance of the hair was noted at age 6 months, arousing only mild curiosity and no medical attention. The growth of hair was normal, requiring a haircut at age 1 year.

The difficulty in combing the boy's hair met with little concern on the part of the parents, because the father had had similar hair during infancy, which had gradually become 'normal' at about age 5 years. The paternal grandfather and great-grandfather were also known to have had this unusual scalp hair in their infancy. No other family members were affected.

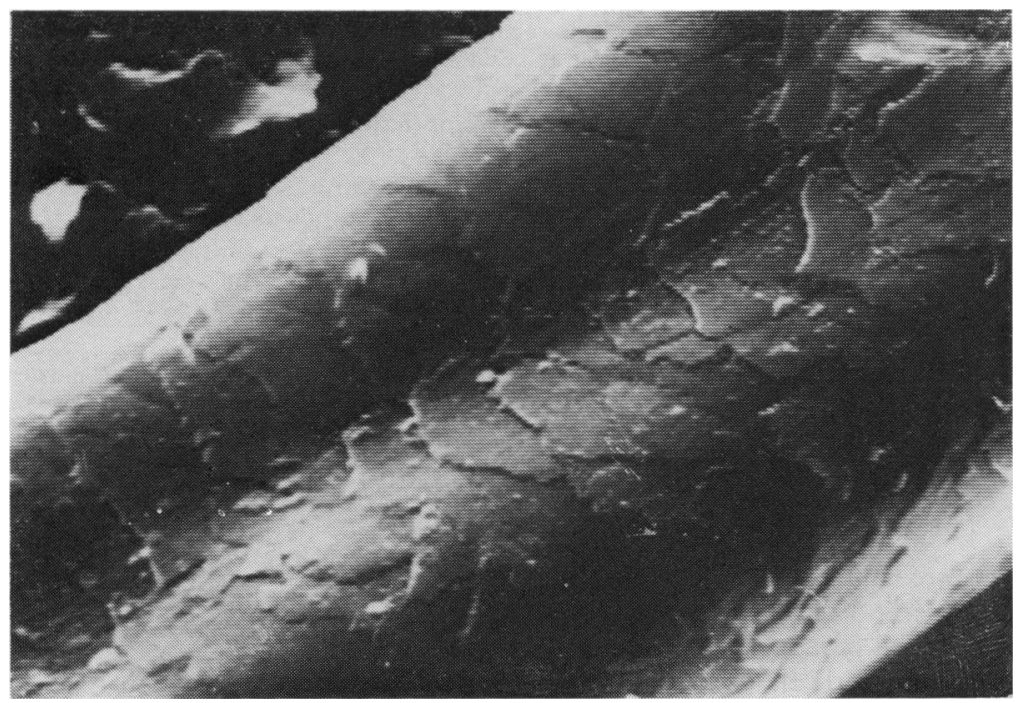

Fig. 2 Scanning electron microscope examination showing longitudinal central groove along the hair long axis. 
Blood count, routine blood chemistry-aminoacids, lipids, iron, copper, zinc, and thyroxine-urine analysis, mucopolysaccharides, and amino-acids in urine, were normal.

The examination of the scalp hair by light microscope showed an uneven scanty distribution of pigment with small pits on the hair shaft and slight twisting of the hair along its axis. Using a scanningelectron microscope, most hair cross-sections were found to have a triangular shape, caused by longitudinal grooves along their axis. The structure and the deposition of the horny lamellae were normal (Fig. 2).

\section{Discussion}

Uncombable hair appears generally during the first year of life and can be considered an anomaly of the terminal hair appearing soon after the lanugo hair is shed. Only rarely does the condition appear later in childhood. ${ }^{3}$ The phenomenon is limited to the scalp hair which is composed of thick bundles of hair which grow in different directions, and cannot be arranged by combing. The hair is dry, generally fair, with a characteristic sheen. It is not fragile and grows normally.

The common morphological features of the anomaly are the presence of one or two longitudinal grooves which are found in the majority of the hairs examined with scanning electron microscope, and on cross-section of the hair it may be triangular or kidney-shaped. However, not all the hairs are abnormal and many morphological variants have been observed. Moreover, these findings are not specific since the appearance of longitudinal grooves along the hair axis was reported in a case of progeria, in monilethrix, and pili torti ${ }^{2}$; a triangular crosssection of the hair was reported also in mucopolysaccharidosis and in normal individuals. ${ }^{4}$

The principal differential diagnosis of 'uncombable hair' is 'woolly hair'. The characteristic of 'woolly hair' is its tightly coiled spirals which do not form locks. The cross-section of woolly hair was found by some to be oval, while the straight hairs are generally round. ${ }^{5}$ According to others, these differences might also be caused by different haircutting techniques. 'Uncombable hair' should also be distinguished from 'unruly hair', which is often associated with mental retardation and microcephaly, but may be present in $2 \%$ of normal infants. ${ }^{6}$

The mode of inheritance of 'uncombable hair' is not known. A familial occurrence was reported in 12 of the 27 cases, including this one. The data on the familial cases are not sufficiently reported; the abnormality was found in 3 out of 8 siblings (the parents being second-cousins), ${ }^{7}$ in father and son, ${ }^{8}$ and in mother and son. ${ }^{2}$ The sex ratio of the reported cases is 12 boys and 15 girls. The almost equal ratio of males and females and the vertical mode of inheritance, especially in our case, in which the abnormality occurred in four consecutive generations, suggests an autosomal dominant mode of inheritance.

In many cases spontaneous amelioration of the condition has occurred during childhood, as in the ancestors of the reported case. The recognition of this entity and the knowledge of its resultant improvement may be helpful for anxious parents if confronted by this unusual hair appearance in their children.

We thank Professor M Djaldetti for the scanning electron microscope examination.

\section{References}

1 Stroud J D, Mehregan A H. 'Spun glass' hair: a clinicopathologic study of an unusual hair defect. In: Brown A, ed. The First Human Hair Symposium. New York: Medcom Press, 1973: 103-7.

2 Dupré A, Bonafé J L, Litoux F, Victor M. Le syndrome des cheveux incoiffables-pili trianguli et canaliculi. Ann Dermatol Venereol 1978; 105: 627-30.

${ }^{3}$ Ferrando J, Fontarnau R, Gratacos M R, Mascaro J M. Pili canaliculi (cheveux incoiffables' ou 'cheveux en fibre de verre'). Dix nouveaux cas avec étude au microscope électronique à balayage. Ann Dermatol Venereol 1980; 107: 243-8.

4 Crump A I, Danks D M. Simple method for cutting transverse sections of hair: comments on shape of hair in Hurler and Sanfilippo syndromes. Arch Dis Child 1971 46: 383-6.

5 Lantis S D H, Pepper M C. Woolly hair nevus. Arch Dermatol 1978; 114: 233-8.

6 Smith D W, Greely M J. Unruly scalp hair in infancy: its nature and relevance to problems of brain morphogenesis. Pediatrics 1978; 61 : 783-5.

7 Grupper C, Attal C, Gougne B. Syndrome des cheveux incoiffables. Bull Soc Fr Dermatol Syph 1974; 81: 299300 .

${ }^{8}$ Laurent R, Yulzari M, Makki S, Agache P. Syndrome des cheveux incoiffables. Deux nouveaux cas familiaux avec étude au microscope électronique à balayage. Ann Dermatol Venereol 1978; 105: 633-5.

Correspondence to Dr B Garty, Department of Paediatrics 'B', Beilinson Medical Centre, 76100 Petah-Tiqva, Israel.

Received 9 February 1982 